\title{
Endoscopic percutaneous suturing (eFe technique) in massive bleeding due to percutaneous endoscopic gastrostomy
}

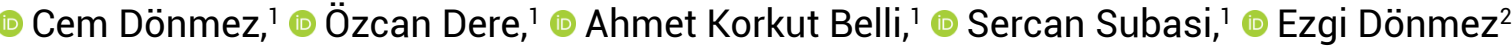 \\ 'Department of General Surgery, Muğla Sıtkı Koçman University Faculty of Medicine, Muğla, Turkey \\ ${ }^{2}$ Department of Anesthesiology and Reanimation, Yatağan State Hospital, Muğla, Turkey
}

\begin{abstract}
Percutaneous endoscopic gastrostomy (PEG) has so many complications, such as haemorrhage and gastrocutaneous fistula, and can be treated with several common procedures. When the common procedures are not successful, we must perform new procedures, such as eFe technique. We use this technique for gastrostomy tube removal at the same session to prevent fistula formation in our clinical practice. Sometimes, leakage beside the gastrostomy tube can be seen during feeding from PEG; we also treat this problem with the same eFe technique in our clinic. We used this technique for the treatment of hemorrhage due to PEG for the first time when could not control with routine procedures and became successful.
\end{abstract}

Keywords: Gastrocutaneous fistula; haemorrhage; laparoscopy; percutaneous endoscopic gastrostomy; trochar.

\section{Introduction}

Percutaneous endoscopic gastrostomy (PEG) is usually performed for long term enteral nutrition when oral intake is inadequate or impossible. ${ }^{[1-3]}$ Of course there are many complications due to PEG such as; haemorrhage, organ damage, aspiration of the stomach content, gastrocutaneous fistula...etc. ${ }^{[4-7]}$ Endoscopic clips, electrocoagulation, scleroterapy are commonly used procedures to control massive hemorhage ${ }^{[8,9]}$ but they may not always be successful. When these common procedures are not successful we have to perform new procedures such as eFe technique.

\section{Which Technique?}

We use Endoscopic Figure of Eight (eFe) technique for gastrostomy tube removal at the same session to prevent fistula formation in our clinical practice. Sometimes leakage beside the gastrostomy tube can be seen during feeding from PEG, we also treat this problem with the same eFe technique in our clinic. Informed consent about the operations was read to the participants and we used this technique for the treatment of hemorrhage due to PEG for the first time when couldn't control with routine procedures and became successful.

A 71-year-old man required PEG due to inadequate oral intake when he was receiving treatment for Amyotrofic Lateral Sclerosis (ALS) and aspiration pneumonia in intensive care unit. Percutaneous endoscopic gastrostomy was performed by other clinic 3 days ago. The patient was 
transferred to our clinic with melena, haemorrhage from gastrostomy tube and hypotension. Hemoglobin level was $6 \mathrm{gr} / \mathrm{dl}$ and he was in hemorrhagic shock. In physical examination he had epigastric sensitivity and gastrostomy tube level was inside the stomach more than necessary. Active haemorrhage was seen beside and through the tube. We retracted the tube in a way to elevate the abdomen for taking the advantage of the pressure of the tube's knob. Blood transfusion was started and bedside graphy and focused abdominal sonography for trauma (FAST) was performed to exclude any leakage into intraabdominal space. There was no intraandominal pathology. The stomach was full of hematoma. When hemodynamics were stabilized, we started endoscopy. We found active arterial haemorrhage (near the corpus-antrum junction from the anterior gastric wall at the entrance site) profoundly from the stomach wall by loosening the gastrostomy tube. We tried endoscopic clips and scleroterapy but couldn't control the haemorrhage. Then we decided to use percutaneous suture needle which we use routinely for bleeding from the trochar entry in laparoscopy. We sutured the bleeding wound with laparoscopic percutaneous fascia closer (Easy close; Atak medical, Istanbul, Turkey) by using endoscopic biopsy forceps (Fig. 1a-d). We were sure that bleeding was controlled by aspirating the air in the stomach. We didn't need to cancel the gastrostomy. We started refeeding from the same gastrostomy tube after 12 hours. There was no problem in 7 days follow-up. We use the same technique routinely for haemorrhages from trochar entry and after gastrostomy tube removal to prevent gastrocutaneous fistula formation. According to us, this procedure is alternative, successful, fast and decreasing surgical requirement.

\section{Discussion}

Percutaneous endoscopic gastrostomy is usually performed for long term enteral nutrition when oral intake is inadequate or impossible. ${ }^{[1-3]}$ Although success rates greater than $95 \%$ have been reported for PEG, procedurerelated complications are common such as; haemorrhage, organ damage, aspiration of the stomach content, gastrocutaneous fistula...etc. ${ }^{[4-7]}$ Normally, the location of the peg in the stomach should be to adhere the stomach to the abdominal wall but not to create pressure so much to prevent ischemia but in our case gastrostomy tube level was inside the stomach more than necessary.

Endoscopic clips, electro coagulation, scleroterapy are commonly used tecniques to control massive haemorrhage ${ }^{[8,9]}$ but sometimes we cannot be successful with
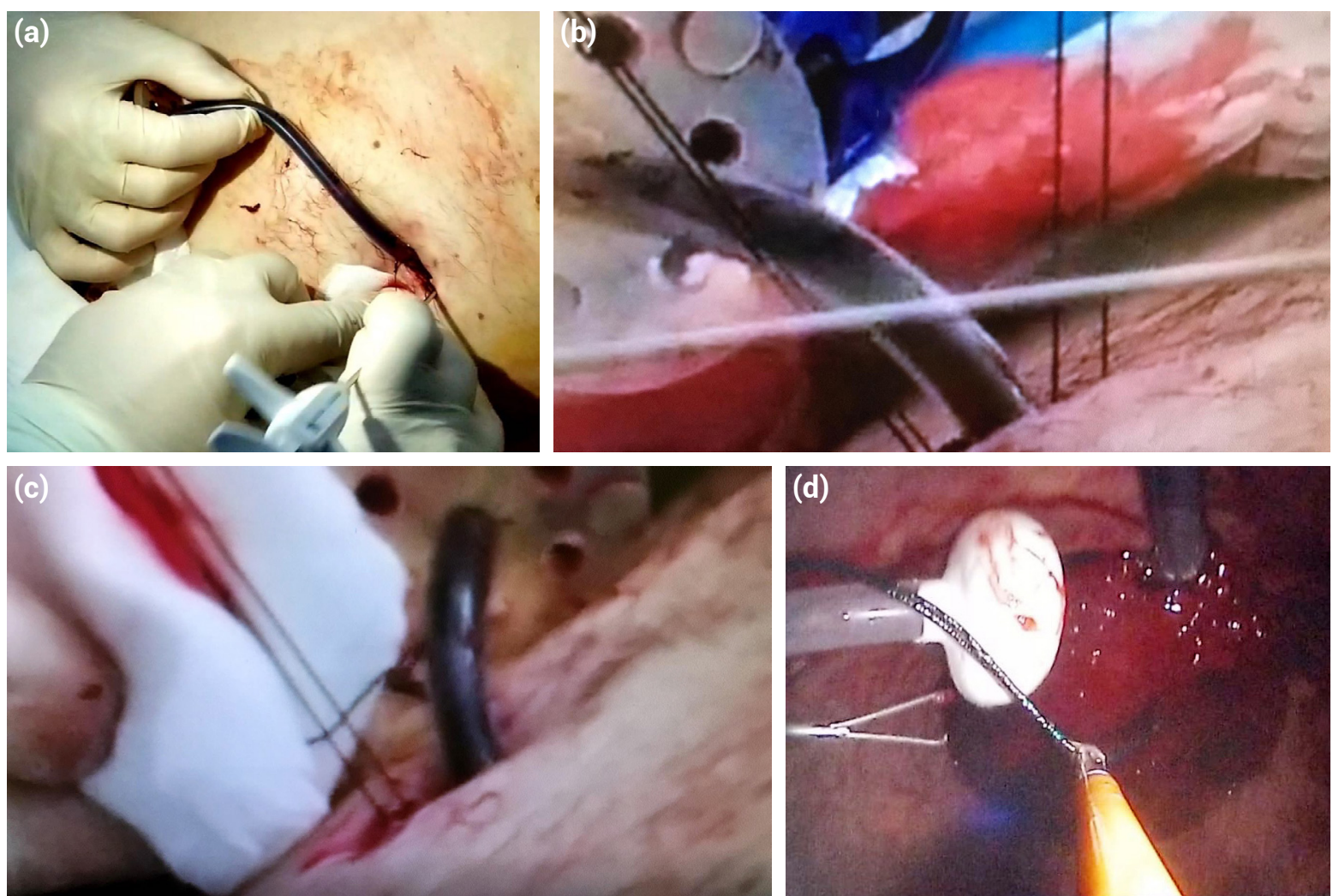

Figure 1. (a) View of the process from outside the abdomen. (b, c) Stitched sutures. (d) Retraction of the suture material catched with biopsy forceps through the endoscop. 
these procedures. We may use this which we call eFe technique, especially for the patients whom bleeding cannot be controlled with these common procedures. We developed eFe technique in the treatment of haemorrhage due to gastrostomy by using laparoscopic percutaneous fascia closer which is routinely used in trocar site bleeding in laparoscopy. eFe technique is easy, cheap, fast, safety, can be feasible at the same session in endoscopy unit and has low mortality and morbidity due to decreasing surgical requirement. A similar technique to this has been performed since 2002 for the treatment of gastrocutaneous fistula formation after gastrostomy tube removal ${ }^{[10]}$ but we didn't find any publication about controlling haemorrhage and preventing gastrocutaneous fistula with this technique in literature review. In the past 2 years we followed 12 patients for 3 months for gastrocutaneous fistula formation after gastrostomy tube removal with this technique and none of them had this problem. We use this technique for gastrostomy tube removal at the same session to prevent fistula formation in our clinical practice. Sometimes leakage beside the gastrostomy tube can be seen during feeding from PEG, we also treat this problem with the same eFe technique in our clinic. We used this technique for the treatment of hemorrhage due to PEG for the first time when couldn't control with routine procedures and became successful. Considering all these positive aspects, eFe technique will be the first we will apply when we encounter peg-related hemorrhage and other peg complications in our clinic.

\section{Conclusion}

eFe technique is gold standard because it is easier, faster and safer in the treatment of most common peg and laparoscopy complications when compared with other techniques according to our clinical experience. We recommend this technique strongly for controlling haemorrhage and also preventing gastrocutaneous fistula formation due to gastrostomy tube removal. This is a simple example of the benefits of surgeons to endoscopy.

\section{Disclosures}

Informed Consent: Written informed consent was obtained from the patient for the publication of the case report and the accompanying images.

Peer-review: Externally peer-reviewed.

Conflict of Interest: None declared.

\section{References}

1. Janik TA, Hendrickson RJ, Janik JS, Landholm AE. Analysis of factors affecting the spontaneous closure of a gastrocutaneous fistula. J Pediatr Surg 2004;39:1197-9. [CrossRef]

2. Kobak GE, McClenathan DT, Schurman SJ. Complications of removing percutaneous endoscopic gastrostomy tubes in children. J Pediatr Gastroenterol Nutr 2000;30:404-7. [CrossRef]

3. Löser C, Aschl G, Hébuterne $X$, Mathus-Vliegen EM, Muscaritoli $\mathrm{M}, \mathrm{Niv} \mathrm{Y}$, et al. ESPEN guidelines on artificial enteral nutrition-percutaneous endoscopic gastrostomy (PEG). Clin Nutr 2005;24:848-61. [CrossRef]

4. Lau G, Lai SH. Fatal retroperitoneal haemorrhage: an unusual complication of percutaneous endoscopic gastrostomy. Forensic Sci Int. 2001;116:69-75. [CrossRef]

5. Bunai $Y$, Akaza K, Nagai A, Tsujinaka M, Jiang WX. Iatrogenic rupture of the left gastric artery during percutaneous endoscopic gastrostomy. Leg Med (Tokyo) 2009;11 Suppl 1:S538-40. [CrossRef]

6. Ghevariya V, Paleti V, Momeni M, Krishnaiah M, Anand S. Complications associated with percutaneous endoscopic gastrostomy tubes. Ann Longterm Care 2009;17:36-41.

7. Blomberg J, Lagergren J, Martin L, Mattsson F, Lagergren P. Complications after percutaneous endoscopic gastrostomy in a prospective study. Scand J Gastroenterol 2012;47:73742. [CrossRef]

8. Baron TH, Song LM, Ross A, Tokar JL, Irani S, Kozarek RA. Use of an over-the-scope clipping device: multicenter retrospective results of the first U.S. experience (with videos). Gastrointest Endosc 2012;76:202-8. [CrossRef]

9. Singhal S, Changela K, Papafragkakis H, Anand S, Krishnaiah M, Duddempudi S. Over the scope clip: technique and expanding clinical applications. J Clin Gastroenterol 2013;47:749-56.

10. Alberti-Flor JJ. Percutaneous-endoscopic suturing of gastrocutaneous fistula: report of 2 cases. Gastrointest Endosc 2002;56:751-3. [CrossRef] 